PROCEEDINGS OF THE

AMERICAN MATHEMATICAL SOCIETY

Volume 124, Number 7, July 1996

\title{
OBLIQUE PROJECTIONS IN ATOMIC SPACES
}

\author{
AKRAM ALDROUBI
}

(Communicated by Palle E. T. Jorgensen)

Abstract. Let $\mathcal{H}$ be a Hilbert space, $\mathbf{O}$ a unitary operator on $\mathcal{H}$, and $\left\{\phi^{i}\right\}_{i=1, \ldots, r} r$ vectors in $\mathcal{H}$. We construct an atomic subspace $U \subset \mathcal{H}$ :

$$
U=\left\{\sum_{i=1, \ldots, r} \sum_{k \in \mathbf{Z}} c^{i}(k) \mathbf{O}^{k} \phi^{i}: c^{i} \in l^{2}, \forall i=1, \ldots, r\right\} .
$$

We give the necessary and sufficient conditions for $U$ to be a well-defined, closed subspace of $\mathcal{H}$ with $\left\{\mathbf{O}^{k} \phi^{i}\right\}_{i=1, \ldots, r, k \in \mathbf{Z}}$ as its Riesz basis. We then consider the oblique projection $\mathbf{P}_{U \perp V}$ on the space $U\left(\mathbf{O},\left\{\phi_{U}^{i}\right\}_{i=1, \ldots, r}\right)$ in a direction orthogonal to $V\left(\mathbf{O},\left\{\phi_{V}^{i}\right\}_{i=1, \ldots, r}\right)$. We give the necessary and sufficient conditions on $\mathbf{O},\left\{\phi_{U}^{i}\right\}_{i=1, \ldots, r}$, and $\left\{\phi_{V}^{i}\right\}_{i=1, \ldots, r}$ for $\mathbf{P}_{U \perp V}$ to be well defined. The results can be used to construct biorthogonal multiwavelets in various spaces. They can also be used to generalize the Shannon-Whittaker theory on uniform sampling.

\section{INTRODUCTION}

Shift-invariant spaces of $L_{2}(\mathbf{R})$ of the form

$$
U=\left\{\sum_{i=1}^{i=r} \sum_{k \in \mathbf{Z}} c^{i}(k) \phi^{i}(x-k): c^{i} \in l_{2}\right\}
$$

are the building blocks for the multiresolution and wavelet theory $[13,14,15,8,1,4]$. Because of their richness and simple structure, these spaces have long been used in many other areas, such as numerical analysis [16, 6, 18], and sampling theory $[5,3,19,20]$, to name a few. If we replace $L_{2}(\mathbf{R})$ by a complex Hilbert space $\mathcal{H}$, and if we choose $r$ functions $\left\{\phi^{i}\right\}_{i=1, \ldots, r} \subset \mathcal{H}$, we then obtain the abstract space

$$
U\left(\mathbf{O},\left\{\phi^{i}\right\}_{i \in S}\right)=\left\{\sum_{k \in \mathbf{Z}} \mathbf{C}(k) \Phi^{T}(k):=\sum_{i \in S} \sum_{k \in \mathbf{Z}} c^{i}(k) \mathbf{O}^{k} \phi^{i}: c^{i} \in l^{2}, \forall i \in S\right\},
$$

where $\mathbf{O}$ is a unitary operator that replaces the shift operator $\mathbf{O} \phi^{i}(x)=\phi^{i}(x-1)$ in (1.1), and where $S=\{1, \ldots, r\}, \mathbf{C}=\left(c^{1}, \ldots, c^{r}\right)$, and $\Phi^{T}(k)$ is the vector transpose of $\Phi(k):=\left(\mathbf{O}^{k} \phi^{1}, \ldots, \mathbf{O}^{k} \phi^{r}\right)$. Since $U$ is generated from a unitary operator $\mathbf{O}$ and from a set of atoms $\left\{\phi^{i}\right\}_{i \in S}$, we will say that $U$ is an atomic subspace of $\mathcal{H}$.

Received by the editors January 3, 1995.

1991 Mathematics Subject Classification. Primary 41A15, 42C15, 46C99, $47 \mathrm{~B} 37$.

Key words and phrases. Oblique projection, biorthogonal multiwavelet, multiwavelets, unitary operators, Riesz basis. 
By observing the relation between wavelets and wandering subspaces in operator theory, Goodman, Lee, and Tang [11] used atomic spaces to construct an abstract theory of multiresolutions and wavelets in Hilbert spaces. As a result, they developed the theory of multiwavelets in $L_{2}(\mathbf{R})$ (see also $[17,9]$ ).

In this paper, we completely characterize the atomic spaces. We give the necessary and sufficient conditions on $\mathbf{O}$ and $\left\{\phi^{i}\right\}_{i \in S}$ for $U$ to be a well-defined, closed subspace of $\mathcal{H}$, with $\left\{\mathbf{O}^{k} \phi^{i}\right\}_{i \in S, k \in \mathbf{Z}}$ as its Riesz basis. We then relate these results to the computation of the orthogonal projection of a vector $g \in \mathcal{H}$ on the space $U$. This is done in section 2. In section 3, we give the necessary and sufficient conditions on $\mathbf{O},\left\{\phi_{U}^{i}\right\}_{i \in S}$, and $\left\{\phi_{V}^{i}\right\}_{i \in S}$ for the existence of the oblique projection $\mathbf{P}_{U \perp V}$ on the space $U$ in a direction orthogonal to $V$. Our results can be used to extend the biorthogonal wavelet theory of Cohen-Daubechies-Feauveau to the case of biorthogonal multiwavelets of $L_{2}$. Our results can also be used to extend the biorthogonal theory to spaces other than $L_{2}\left(\mathbf{R}^{d}\right)$ and to geometries other than the Euclidian geometry.

\section{ATOMIC SPACES}

The definition of the space $U$ in (1.2) makes sense if there exists a positive number $0<M<\infty$ such that

$$
\left\|\sum_{k \in \mathbf{Z}} \mathbf{C}(k) \Phi^{T}(k)\right\|_{\mathcal{H}}^{2} \leq M\|\mathbf{C}\|_{l_{2}^{r}}^{2} \quad \forall \mathbf{C} \in l_{2}^{r}
$$

where $l_{2}^{r}:=l_{2}(\mathbf{Z}) \times \cdots \times l_{2}(\mathbf{Z})$ and where $\|\mathbf{C}\|_{l_{2}^{r}}^{2}=\sum_{i \in S}\left\|c^{i}\right\|_{l_{2}}^{2}$. If, in addition, we have the lower bound

$$
m\|\mathbf{C}\|_{l_{2}^{r}}^{2} \leq\left\|\sum_{k \in \mathbf{Z}} \mathbf{C}(k) \Phi^{T}(k)\right\|_{\mathcal{H}}^{2},
$$

where $m>0$, then $U$ is a closed subspace of $\mathcal{H}$ and the set $\left\{\mathbf{O}^{k} \phi^{i}\right\}_{i \in S, k \in \mathbf{Z}}$ is a Riesz basis for $U \subset \mathcal{H}$. Our goal is then to find conditions on $\mathbf{O}$ and $\left\{\phi^{i}\right\}_{i \in S}$ for $U$ to be a well-defined subspace of $\mathcal{H}$, and for $\left\{\mathbf{O}^{k} \phi^{i}\right\}_{i \in S, k \in \mathbf{Z}}$ to be its Riesz basis. To do this, we first observe that if $U$ is well defined (i.e., condition (2.1) is satisfied), then we can write the norm of a vector $u=\sum \mathbf{C}(k) \Phi^{T}(k)$ belonging to $U$ as

$$
\|u\|_{\mathcal{H}}^{2}=\sum_{(k, l) \in \mathbf{Z}^{2}} \mathbf{C}(k) \mathbf{A}(l-k) \mathbf{C}^{*}(l)
$$

where the sequence of matrices $\mathbf{A}(k), k \in \mathbf{Z}$, is defined to be

$$
[\mathbf{A}]_{i, j}(k):=\left\langle\phi^{i}, \mathbf{O}^{k} \phi^{j}\right\rangle_{\mathcal{H}} .
$$

Here, $\langle\bullet, \bullet\rangle_{\mathcal{H}}$ denotes the inner product on $\mathcal{H}$.

Using Parseval's theorem and the fact that the convolution operator transforms into the product operator in Fourier domain, (2.3) becomes

$$
\|u\|_{\mathcal{H}}^{2}=\int_{0}^{1} \widehat{\mathbf{C}}(f) \widehat{\mathbf{A}}(f) \widehat{\mathbf{C}}^{*}(f) d f
$$


where the Fourier transform of a sequence $\mathbf{b}(k)$ is defined to be

$$
\widehat{\mathbf{b}}(f)=\sum \mathbf{b}(k) e^{-i 2 \pi k f},
$$

and where $\widehat{\mathbf{C}}^{*}$ denotes the Hermitian transpose of $\widehat{\mathbf{C}}$. From this definition, it follows that $\widehat{\mathbf{A}}(f)$ is 1-periodic (i.e., $\widehat{\mathbf{A}}(f+1)=\widehat{\mathbf{A}}(f))$. Moreover, the $r \times r$ matrix $\widehat{\mathbf{A}}(f)$ in (2.4) is self-adjoint for almost all $f$. To see this, we use the fact that $\mathbf{O}$ is unitary, and simply note that

$$
\begin{aligned}
{[\widehat{\mathbf{A}}(f)]_{i, j} } & =\left\langle\phi^{i}, \phi^{j}\right\rangle_{\mathcal{H}}+\sum_{k=1}^{k=\infty}\left\langle\phi^{i}, \mathbf{O}^{k} \phi^{j}\right\rangle_{\mathcal{H}} e^{-i 2 \pi f k}+\left\langle\phi^{i}, \mathbf{O}^{-k} \phi^{j}\right\rangle_{\mathcal{H}} e^{i 2 \pi f k} \\
& =\left\langle\phi^{i}, \phi^{j}\right\rangle_{\mathcal{H}}+\sum_{k=1}^{k=\infty}\left\langle\mathbf{O}^{-k} \phi^{i}, \phi^{j}\right\rangle_{\mathcal{H}} e^{-i 2 \pi f k}+\left\langle\mathbf{O}^{k} \phi^{i}, \phi^{j}\right\rangle_{\mathcal{H}} e^{i 2 \pi f k} \\
& =\overline{\left\langle\phi^{j}, \phi^{i}\right\rangle_{\mathcal{H}}}+\sum_{k=1}^{k=\infty} \overline{\left\langle\phi^{j}, \mathbf{O}^{-k} \phi^{i}\right\rangle_{\mathcal{H}} e^{i 2 \pi f k}}+\overline{\left\langle\phi^{j}, \mathbf{O}^{k} \phi^{i}\right\rangle_{\mathcal{H}} e^{-i 2 \pi f k}} \\
& =\overline{[\widehat{\mathbf{A}}(f)]_{j, i}}
\end{aligned}
$$

where we use $\bar{z}$ to denote the complex conjugate of $z$. From (2.4), it also follows that $\widehat{\mathbf{A}}(f)$ is positive. In fact, we have:

Theorem 2.1. The space $U$ is a well-defined, closed subspace of $\mathcal{H}$ with Riesz basis $\left\{\mathbf{O}^{k} \phi^{i}\right\}_{i \in S, k \in \mathbf{Z}}$ if and only if the $r \times r$ matrix $\widehat{\mathbf{A}}(f)$ is positive self-adjoint for almost all $f \in \mathbf{R}$ and there exist two positive constants $0<m \leq M<\infty$ such that the smallest and largest eigenvalues $\lambda_{\min }(\widehat{\mathbf{A}}(f))$ and $\lambda_{\max }(\widehat{\mathbf{A}}(f))$ satisfy:

$$
m \leq \operatorname{ess}_{f \in\left[-\frac{1}{2}, \frac{1}{2}\right]}\left(\lambda_{\min }(\widehat{\mathbf{A}}(f))\right) \leq \operatorname{ess~sup}_{f \in\left[-\frac{1}{2}, \frac{1}{2}\right]}\left(\lambda_{\max }(\widehat{\mathbf{A}}(f))\right) \leq M \quad \text { a.e. }
$$

We should note that our definition of atomic spaces excludes finite-dimensional spaces. For related results in finite dimensions, we refer the reader to [10]. Other related results can also be found in [12].

Proof. Necessity. If conditions (2.1) and (2.2) hold, then-as we have shown above$\widehat{\mathbf{A}}(f)$ is a 1-periodic and positive self-adjoint matrix for almost all $f$. Thus, it has $r$ real eigenvalues. If we assume that the largest eigenvalue $\lambda_{\max }(f)$ is not bounded above by $M$, then the set $E_{n}:=\left\{f \in\left[-\frac{1}{2}, \frac{1}{2}\right]: \lambda_{\max }(f)>n\right\}$ has a strictly positive measure: meas $\left(E_{n}\right)>0$ (we only need to concentrate on the interval $\left[-\frac{1}{2}, \frac{1}{2}\right]$ because $\lambda_{\max }(f)$ is 1-periodic). We consider the periodic vector functions $\widehat{\mathbf{C}}_{n}(f+1)=\widehat{\mathbf{C}}_{n}(f)$ defined by $\widehat{\mathbf{C}}_{n}(f):=\left(\operatorname{meas}\left(E_{n}\right)\right)^{-1 / 2} \chi_{E_{n}} \mathbf{V}_{\lambda_{\max }}(f)$ for all $f \in\left[-\frac{1}{2}, \frac{1}{2}\right]$, where $\chi_{E_{n}}$ is the characteristic function of the set $E_{n}$ and $\mathbf{V}_{\lambda_{\max }}(f)$ is the eigenvector corresponding to $\lambda_{\max }(f)$ with $\left\|\mathbf{V}_{\lambda_{\max }}(f)\right\|_{\mathbf{R}^{r}}=1$. By construction, $\widehat{\mathbf{C}}_{n}(f)$ are the Fourier transforms of vector sequences $\mathbf{C}_{n}(k)$ in $l_{2}^{r}$ with $\left\|\mathbf{C}_{n}\right\|_{l_{2}^{r}}=1$. Using identity (2.4), and the fact that $\left\|\mathbf{C}_{n}\right\|_{l_{2}^{r}}^{2}=1$, we conclude that the norm of the vectors $u_{n}=\sum_{k \in \mathbf{Z}} \mathbf{C}_{n}(k) \Phi^{T}(k)$ tends to infinity as $n$ goes to infinity: $\left\|u_{n}\right\|_{\mathcal{H}}^{2} \geq n$. This contradicts our hypothesis that $\left\|u_{n}\right\|_{\mathcal{H}}^{2} \leq M$. Thus, 
$\lambda_{\max }(f) \leq M$ almost everywhere. A similar argument using the smallest eigenvalue $\lambda_{\text {min }}(f)$ implies that $\lambda_{\min }(f)$ is bounded below by a positive constant $m$.

Sufficiency. If $\widehat{A}(f)$ is positive self-adjoint for almost all $f$, then from (2.4) we obtain

$$
\int_{0}^{1} \lambda_{\min }(f) \sum_{i \in S}\left|\hat{c}^{i}(f)\right|^{2} d f \leq \int_{0}^{1} \widehat{\mathbf{C}}(f) \widehat{\mathbf{A}}(f) \widehat{\mathbf{C}}^{*}(f) d f \leq \int_{0}^{1} \lambda_{\max }(f) \sum_{i \in S}\left|\hat{c}^{i}(f)\right|^{2} d f .
$$

Condition (2.6) and the properties of the Fourier transform then imply that

$$
m\|\mathbf{C}\|_{l_{2}^{r}}^{2} \leq\left\|\sum_{k \in \mathbf{Z}} \mathbf{C}(k) \Phi^{T}(k)\right\|_{\mathcal{H}}^{2} \leq M\|\mathbf{C}\|_{l_{2}^{r}}^{2} .
$$

Since $\widehat{\mathbf{A}}(f)$ is positive and self-adjoint, we have that $\lambda_{\max }(f)=\|\widehat{\mathbf{A}}(f)\|$ and that $\left(\lambda_{\min }(f)\right)^{-1}=\left\|\widehat{\mathbf{A}}^{-1}(f)\right\|$ where $\|\bullet\|$ denotes the norm of the operators on $\mathbf{R}^{r}$. Thus, condition (2.6) is equivalent to the conditions $\|\widehat{\mathbf{A}}(f)\| \leq M$ and $\left\|\widehat{\mathbf{A}}^{-1}(f)\right\| \leq$ $m^{-1}$ almost everywhere. These two conditions imply that the convolution operator - $* \mathbf{A}(k)$ which takes a sequence $\mathbf{C}(k)$ into the sequence $\mathbf{C} * \mathbf{A}(k)=\sum_{l \in \mathbf{Z}} \mathbf{C}(l) \mathbf{A}(k-l)$, is a bounded linear operator on $l_{2}^{r}$ and has a bounded convolution inverse. This last assertion is an immediate consequence of the following theorem:

Theorem 2.2. The convolution operator $\bullet * \mathbf{X}(k)$, generated from the $r \times r$ matrixsequence $\mathbf{X}(k)$, is a bounded linear operator on $l_{2}^{r}$ if and only if there exists a positive constant $M$ such that

$$
\|\widehat{\mathbf{X}}(f)\|^{2}=\left\|\widehat{\mathbf{X}}(f) \widehat{\mathbf{X}}^{*}(f)\right\| \leq M<\infty \text { a.e. }
$$

Moreover, the norm of the convolution operator is given by

$$
\|\bullet * \mathbf{X}\|_{B\left(l_{2}^{r}\right)}=\underset{f \in[0,1]}{\operatorname{ess} \sup _{1}}\|\widehat{\mathbf{X}}(f)\| .
$$

Proof. With an argument similar to the one that led to (2.4), we get

$$
\|\mathbf{C} * \mathbf{X}\|_{l_{2}^{r}}^{2} \leq \int_{0}^{1} \lambda_{\max }(f) \widehat{\mathbf{C}}(f) \widehat{\mathbf{C}}^{*}(f) d f \leq \Lambda \int_{0}^{1} \widehat{\mathbf{C}}(f) \widehat{\mathbf{C}}^{*}(f) d f
$$

where $\lambda_{\max }(f)$ is the largest eigenvalue of the $r \times r$ self-adjoint matrix $\widehat{\mathbf{X}}(f) \widehat{\mathbf{X}}^{*}(f)$, and where $\Lambda:=\operatorname{ess~sup}_{f \in[0,1]}\left(\lambda_{\max }(f)\right)$. It follows that $\|\bullet * \mathbf{X}\|_{B\left(l_{2}^{r}\right)} \leq \Lambda^{1 / 2}$. Conversely, using a proof by contradiction similar to the "necessity" proof of Theorem 2.1, we get that $\Lambda^{1 / 2} \leq\|\bullet * \mathbf{X}\|_{B\left(l_{2}^{r}\right)}$. The proof of the theorem then follows by simply noting that $\|\widehat{\mathbf{X}}(f)\|^{2}=\left\|\widehat{\mathbf{X}}(f) \widehat{\mathbf{X}}^{*}(f)\right\|=\lambda_{\max }(f)$.

As a consequence of Theorem 2.2, we also conclude that the inverse Fourier transform of $\widehat{\mathbf{A}}^{i n v}(f):=\widehat{\mathbf{A}}^{-1}(f)$ makes sense, and that $\mathbf{A}^{i n v}(k)$ is the convolution inverse of $\mathbf{A}(k)$ :

$$
\sum_{k \in \mathbf{Z}} \mathbf{A}(k) \mathbf{A}^{i n v}(l-k)=\delta(k) \mathbf{I},
$$

where $\mathbf{I}$ is the $r \times r$ identity matrix and where $\delta(k)$ is the Dirac impulse (i.e., $\delta(0)=1$ and $\delta(k)=0$ for $k \neq 0)$. 
If we assume that $\left\|\phi^{i}\right\|_{\mathcal{H}}=1$, then the sequences $[\mathbf{A}]_{i, j}(k)=\left\langle\phi^{i}, \mathbf{O}^{k} \phi^{j}\right\rangle_{\mathcal{H}}$ have the property that $\left|[\mathbf{A}]_{i, i}(0)\right|=1$ and $\left|[\mathbf{A}]_{i, j}(k)\right| \leq 1$ for $k \neq 0, i \neq j$. For this reason, we will call $\mathbf{A}(k)$ the autocorrelation matrix-sequence. In fact, if $\mathcal{H}=L_{2}(\mathbf{R})$, if $r=$ 1 , and if $\mathbf{O}$ is the shift operator, then $\mathbf{A}(k)$ is precisely the sampled autocorrelation function $\mathbf{A}(k)=\int_{\mathbf{R}} \phi(x) \phi(x-k) d x$.

2.1. Orthogonal projection. If the autcorrelation sequence $\mathbf{A}(k)$ satisfies condition (2.6), then $U$ is closed. Thus, the orthogonal projection is well defined. In fact, if $\mathbf{P} g=\sum \mathbf{E}(k) \Phi^{T}(k)$ is the orthogonal projection of $g \in \mathcal{H}$, then from the orthogonality condition $\left\langle\mathbf{P} g-g, \mathbf{O}^{l} \phi^{j}\right\rangle_{\mathcal{H}}=0$, we get the convolution equation

$$
\sum_{k \in \mathbf{Z}} \mathbf{E}(k) \mathbf{A}(l-k)=\mathbf{G}(l) \quad \forall l \in \mathbf{Z},
$$

where $\mathbf{G}$ is the $1 \times r$ vector-sequence $[\mathbf{G}]_{j}(l):=\left\langle g, \mathbf{O}^{l} \phi^{j}\right\rangle_{\mathcal{H}}$. Equation (2.9) allows us to write the orthogonal projection as

$$
\mathbf{P} g=\sum_{k \in \mathbf{Z}} \mathbf{E}(k) \Phi^{T}(k)=\sum_{(k, l) \in \mathbf{Z}^{2}} \mathbf{G}(l) \mathbf{A}^{i n v}(k-l) \Phi^{T}(k)
$$

where, as before, $\mathbf{A}^{i n v}(k)$ is the inverse Fourier transform of $\widehat{\mathbf{A}}^{-1}(f)$, and $\Phi^{T}(k)$ is the vector-transpose of $\Phi(k):=\left(\mathbf{O}^{k} \phi^{1}, \ldots, \mathbf{O}^{k} \phi^{r}\right)$. Because of the simple atomic structure of $U$, all the operations involved in computing the vector-sequence $\mathbf{E}(k)$ in (2.9) consist of simple convolutions and additions only. This feature is often useful for signal processing and numerical analysis because it allows for fast "filtering" implementations.

\section{Oblique projections}

Let $U\left(\mathbf{O},\left\{\phi_{U}^{i}\right\}_{i \in S}\right)$ and $V\left(\mathbf{O},\left\{\phi_{V}^{i}\right\}_{i \in S}\right)$ be two atomic subspaces of $\mathcal{H}$ that satisfy condition (2.6). Then, under appropriate conditions, the oblique projection $\mathbf{P}_{U \perp V} g=\sum \mathbf{C}_{g}(k) \Phi_{U}^{T}(k)$ of a vector $g \in \mathcal{H}$ on the space $U$, in a direction orthogonal to $V$, must satisfy $\left\langle\mathbf{P}_{U \perp V} g-g, \mathbf{O}^{l} \phi_{V}^{j}\right\rangle_{\mathcal{H}}=0$ for all $j \in S$ and for all $l \in \mathbf{Z}$. From this, we obtain the convolution equation

$$
\sum_{k \in \mathbf{Z}} \mathbf{C}_{g}(k) \mathbf{X}_{U V}(l-k)=\mathbf{G}(l),
$$

where $\mathbf{X}_{U V}$ is the cross-correlation matrix-sequence $\left[\mathbf{X}_{U V}\right]_{i, j}(k)=\left\langle\phi_{U}^{i}, \mathbf{O}^{k} \phi_{V}^{j}\right\rangle_{\mathcal{H}}$ and where $\mathbf{G}$ is the $1 \times r$ vector given by $[\mathbf{G}]_{j}(l)=\left\langle g, \mathbf{O}^{l} \phi_{V}^{j}\right\rangle_{\mathcal{H}}$. In the Fourier domain, (3.1) becomes

$$
\widehat{\mathbf{C}}_{g}(f) \widehat{\mathbf{X}}_{U V}(f)=\widehat{\mathbf{G}}(f) .
$$

Our first observation is that the convolution operator $\bullet * \mathbf{X}_{U V}$ is a bounded operator on $l_{2}^{r}$, a fact solely based on our assumptions that $U$ and $V$ satisfy condition (2.6). To see why our last assertion is true, we pick a vector $g=\sum \mathbf{D}(k) \Phi_{U}^{T}(k)$ in the space $U$ and compute the orthogonal projection $\mathbf{P}_{V} g$ of the vector $g \in U$ on the 
space $V$. A simple calculation shows that the sequence $\mathbf{G}$ in (2.9) is given by $\mathbf{G}=\mathbf{D} * \mathbf{X}_{U V}$. Using eqs. (2.1), (2.2), (2.9), and Theorem 2.1, we get that

$$
\begin{aligned}
\left\|\mathbf{D} * \mathbf{X}_{U V}\right\|_{l_{2}^{r}}^{2}=\left\|\mathbf{E} * \mathbf{A}_{V}\right\|_{l_{2}^{r}}^{2} \leq M_{V}\|\mathbf{E}\|_{l_{2}^{r}}^{2} & \leq m_{V}^{-1} M_{V}\left\|\mathbf{P}_{V} g\right\|_{\mathcal{H}}^{2} \\
& \leq m_{V}^{-1} M_{V}\|g\|_{\mathcal{H}}^{2} \leq m_{U}^{-1} m_{V}^{-1} M_{V}\|\mathbf{D}\|_{l_{2}^{r}}^{2}
\end{aligned}
$$

where $\mathbf{A}_{V}$ is the autocorrelation operator $\mathbf{A}$ in Theorem 2.1 associated with the space $V\left(\mathbf{O},\left\{\phi_{V}^{i}\right\}_{i \in S}\right)$, and $m_{V}, M_{V}$ are the corresponding constants in (2.6), while the constant $m_{U}$ relates to the space $U$. From (3.3), we conclude that $\bullet * \mathbf{X}_{U V}$ is a bounded convolution operator on $l_{2}^{r}$. Equivalently, as a consequence of Theorem 2.2 , there exists a constant $M$ such that, except possibly on a set of measure zero, $\left\|\widehat{\mathbf{X}}_{U V}(f)\right\| \leq M<\infty$. If, furthermore, we have that $\left\|\widehat{\mathbf{X}}_{U V}^{-1}(f)\right\| \leq m<\infty$, then the oblique projection $\mathbf{P}_{U \perp V}$ is well defined. ${ }^{1}$ The converse is also true, and we have:

Theorem 3.1. Let $U\left(\mathbf{O},\left\{\phi_{U}^{i}\right\}_{i \in S}\right)$ and $V\left(\mathbf{O},\left\{\phi_{V}^{i}\right\}_{i \in S}\right)$ be two atomic subspaces of $\mathcal{H}$ that satisfy condition (2.6). The oblique projection $\mathbf{P}_{U \perp V}$ is well defined if and only if $\widehat{\mathbf{X}}_{U V}^{-1}(f)$ exists for almost all $f \in[0,1]$, and if there exists a constant $m>0$ such that

$$
\left\|\widehat{\mathbf{X}}_{U V}^{-1}(f)\right\| \leq m<\infty
$$

The proof of Theorem 3.1 is given below. This theorem can be restated in terms of an "angle" between the spaces $U$ and $V$. In particular, we define the angle $\theta(U, V)$ between the spaces $U$ and $V$ by

$$
\cos (\theta(U, V)):=\inf _{\substack{u \in U \\\|u\|_{\mathcal{H}}=1}}\left\|\mathbf{P}_{V} u\right\|_{\mathcal{H}}
$$

Although $\theta(U, V)=\theta(V, U)$ for the atomic spaces $U$ and $V$ (as we will see below), this is not true for arbitrary spaces. We have:

Theorem 3.2. If the atomic spaces $U\left(\mathbf{O},\left\{\phi_{U}^{i}\right\}_{i \in S}\right)$ and $V\left(\mathbf{O},\left\{\phi_{V}^{i}\right\}_{i \in S}\right)$ satisfy condition (2.6), then

(i) $\cos (\theta(U, V))=\cos (\theta(V, U))=\underset{f \in[0,1]}{\operatorname{ess} \inf }\left(\lambda_{\min }\left(\widehat{\Xi}_{U V}(f)\right)\right)$, where $\widehat{\Xi}_{U V}(f)$ is defined by (3.10) below.

(ii) $\cos (\theta(U, V))=\cos \left(\theta\left(V^{\perp}, U^{\perp}\right)\right)$.

(iii) The oblique projection $\mathbf{P}_{U \perp V}$ is well defined if and only if $\cos (\theta(U, V))>0$.

Remark 3.1. The identity (ii) in Theorem 3.2 holds even if $U$ and $V$ are not atomic spaces.

Remark 3.2. We would expect a condition similar to the one in (iii) for the existence of $\mathbf{P}_{U \perp V}$ to be necessary, in general. In this case, the condition is also sufficient.

Proof of Theorem 3.1. Necessity. Our goal is to find a solution to the convolution equation

$$
\mathbf{C} * \mathbf{X}_{U V}=\mathbf{B}
$$

\footnotetext{
${ }^{1}$ The oblique projection $\mathbf{P}_{U \perp V}$ is well-defined if and only if, for each vector $g \in \mathcal{H}$, there exits a unique representation of the form $g=u_{1}+v_{1}$ with $u_{1} \in U$ and $v_{1} \in V^{\perp}$.
} 
where $\mathbf{B}$ is an arbitrary vector-sequence of $l_{2}^{r}$. To find a solution $\mathbf{C}$, we construct the vector $g \in V$, given by

$$
g=\sum_{k \in \mathbf{Z}}\left(\mathbf{B} * \mathbf{A}_{V}^{i n v}\right)(k) \Phi_{V}^{T}(k)
$$

where $\mathbf{A}_{V}^{i n v}$ is the inverse of the autocorrelation matrix-sequence $\mathbf{A}_{V}(k)$. Since we are assuming that the oblique projection is well defined, we construct the vector $\mathbf{P}_{U \perp V} g=\sum \mathbf{C}_{g}(k) \Phi_{U}^{T}(k)$. A simple calculation using the fact that

$$
\left\langle\mathbf{P}_{U \perp V} g-g, \mathbf{O}^{l} \phi_{V}^{j}\right\rangle_{\mathcal{H}}=0
$$

shows that $\mathbf{C}_{g} * \mathbf{X}_{U V}=\mathbf{B}$. Moreover, $\mathbf{C}_{g}$ is the only solution to (3.6). To see why this claim is true, we assume that there exits a nonzero sequence $\mathbf{C} \in l_{2}^{r}$ such that $\mathbf{C} * \mathbf{X}_{U V}=0$. A simple calculation then shows that the nonzero vector $u=\sum \mathbf{C}(k) \Phi_{U}^{T}(k)$ is orthogonal to $V$. Using the fact that $\mathbf{P}_{U \perp V}$ is well defined, we get that $\mathbf{P}_{U \perp V} u=0$. But since $u \in U$, we also get that $\mathbf{P}_{U \perp V} u=u$. Hence, we deduce that $u=0$, a fact that contradicts our assumption that $\mathbf{C} \neq 0$. Therefore the only solution to the homogeneous equation is $\mathbf{C}=0$. It follows that our constructed solution $\mathbf{C}_{g}$ is unique, and the operator $\bullet * \mathbf{X}_{U V}$ has an inverse. From a calculation similar to (3.3) and from Theorem 2.2, we then conclude that (3.4) holds.

Sufficiency. If $g \in \mathcal{H}$, then the sequence $[\mathbf{G}]_{j}(l)=\left\langle g, \mathbf{O}^{l} \phi_{V}^{j}\right\rangle_{\mathcal{H}}$ is given by

$$
\left\langle g, \mathbf{O}^{l} \phi_{V}^{j}\right\rangle_{\mathcal{H}}=\left\langle g_{V}, \mathbf{O}^{l} \phi_{V}^{j}\right\rangle_{\mathcal{H}}=\sum_{k \in \mathbf{Z}} \mathbf{D}(k) \mathbf{A}_{V}(l-k),
$$

where $g_{V}=\sum_{k \in \mathbf{Z}} \mathbf{D}(k) \Phi_{V}^{T}(k)$ is the orthogonal projection of $g \in \mathcal{H}$ on $V$. Since by assumption $\mathbf{A}_{V}$ satisfies condition (2.6), Theorem 2.2 then implies that $\mathbf{G} \in l_{2}^{r}$. If we assume that condition (3.4) holds, then from Theorem 2.2 it follows that $\mathbf{C}_{g}=\mathbf{G} * \mathbf{X}_{U V}^{-1}$ belongs to $l_{2}^{r}$. It is not difficult to see that the vector $u_{g}=$ $\sum \mathbf{C}_{g}(k) \Phi_{U}^{T}(k)$ belongs to $U$ and that the vector $e_{g}=g-u_{g}$ is orthogonal to $V$. Moreover, the decomposition $g=u_{g}+e_{g}$ is unique. This follows from the fact that if $u=\sum \mathbf{C}(k) \Phi_{U}^{T}(k)$ is orthogonal to $V$, then (from $\left\langle u, \mathbf{O}^{l} \phi_{V}^{j}\right\rangle_{\mathcal{H}}=0$ ) we get $\mathbf{C} * \mathbf{X}_{U V}=0$. The last equality implies that $\mathbf{C}=0$ by assumption. Thus, $u=0$. It follows that the only vector $u \in U$ which is orthogonal to $V$ is the trivial vector (i.e., $U \cap V^{\perp}=\{0\}$ ). From this, we conclude that the decomposition of $g$ into components in $U$ and $V^{\perp}$ is unique.

Proof of Theorem 3.2. If $\mathbf{P}_{V} u=\sum \mathbf{E}(k) \Phi_{V}^{T}(k)$ is the orthogonal projection of the vector $u=\sum \mathbf{D}(k) \Phi_{U}^{T}(k) \in U$ on the space $V$, then by a simple calculation that uses the results of section 2.1, we obtain the relation

$$
\widehat{\mathbf{E}}(f)=\widehat{\mathbf{D}}(f) \widehat{\mathbf{X}}_{U V}(f) \widehat{\mathbf{A}}_{V}^{-1}(f) .
$$

Using the above relation and (2.4), and using the superscript $*$ to denote the Hermitian transpose, we obtain

$$
\left\|\mathbf{P}_{V} u\right\|_{\mathcal{H}}^{2}=\int_{0}^{1} \widehat{\mathbf{Y}}(f) \widehat{\Xi}_{U V}(f) \widehat{\mathbf{Y}}^{*}(f) d f,
$$


where $\widehat{\mathbf{Y}}(f):=\widehat{\mathbf{D}}(f) \widehat{\mathbf{A}}^{1 / 2}(f)$ and where $\widehat{\Xi}_{U V}(f)$ is given by

$$
\begin{gathered}
\widehat{\Xi}_{U V}(f)=\widehat{\mathbf{R}}_{U V}(f) \widehat{\mathbf{R}}_{U V}^{*}(f), \\
\widehat{\mathbf{R}}_{U V}(f)=\widehat{\mathbf{A}}_{U}^{-1 / 2}(f) \widehat{\mathbf{X}}_{U V}(f) \widehat{\mathbf{A}}_{V}^{-1 / 2}(f) .
\end{gathered}
$$

From the definition of $\widehat{\mathbf{Y}}(f)$ and from relation (2.4), we also get that

$$
\|u\|_{\mathcal{H}}^{2}=\int_{0}^{1} \widehat{\mathbf{Y}}(f) \widehat{\mathbf{Y}}^{*}(f) d f .
$$

Combining equalities (3.9) and (3.12), and using the fact that $\widehat{\Xi}_{U V}(f)$ is a positive self-adjoint matrix for almost all $f$, we get the lower bound

$$
\rho^{2}\|u\|_{\mathcal{H}}^{2} \leq \int_{0}^{1} \widehat{\mathbf{Y}}(f) \widehat{\Xi}_{U V}(f) \widehat{\mathbf{Y}}^{*}(f) d f
$$

where $\rho=\cos (\theta(U, V))=\underset{f \in\left[-\frac{1}{2}, \frac{1}{2}\right]}{\operatorname{essinf}}\left(\lambda_{\min }(f)\right)$ (here $\lambda_{\min }(f)$ is the smallest eigenvalue of $\left.\widehat{\Xi}_{U V}(f)\right)$. Since $\widehat{\Xi}_{U V}(f)$ is a positive self-adjoint matrix, we conclude from (3.13) that $\widehat{\Xi}_{U V}(f)$ has an inverse and $\left\|\widehat{\Xi}_{U V}^{-1}(f)\right\|$ is bounded by a constant if and only if the lower bound $\rho>0$. From this last assertion and from eqs. (3.10) and (3.11) and Theorem 3.1, we get part (iii) of Theorem 3.2.

The proof of (ii) can be found in [19]. We show it here for completeness. Let $U^{\perp}$ denote the orthogonal complement of $U$. Using the fact that

$$
\left\|\mathbf{P}_{U} v\right\|_{\mathcal{H}}^{2}+\left\|\mathbf{P}_{U^{\perp}} v\right\|_{\mathcal{H}}^{2}=\|v\|_{\mathcal{H}}^{2}
$$

we get that

$$
\inf _{B_{V}^{1}}\left\|\mathbf{P}_{U} v\right\|_{\mathcal{H}}^{2}=\sup _{B_{V}^{1}}\left\|\mathbf{P}_{U^{\perp}} v\right\|_{\mathcal{H}}^{2}
$$

where $B_{V}^{1}$ is the ball of radius 1 in $V$. Using (3.14) and the general identity

$$
\left\|\mathbf{P}_{W} v\right\|_{\mathcal{H}}=\sup _{B_{W}^{1}}\langle w, v\rangle_{\mathcal{H}}
$$

we obtain

$$
\begin{array}{r}
\inf _{B_{V}^{1}}\left\|\mathbf{P}_{U} v\right\|_{\mathcal{H}}=\sup _{B_{V}^{1}}\left\|\mathbf{P}_{U^{\perp}} v\right\|_{\mathcal{H}}=\sup _{B_{V}^{1}, B_{U^{\perp}}^{1}}\left|\left\langle u^{\perp}, v\right\rangle_{\mathcal{H}}\right| \\
=\sup _{B_{U^{\perp}}^{1}}\left\|\mathbf{P}_{V} u^{\perp}\right\|_{\mathcal{H}}=\inf _{B_{U^{\perp}}^{1}}\left\|\mathbf{P}_{V^{\perp}} u^{\perp}\right\|_{\mathcal{H}}
\end{array}
$$

from which (ii) follows.

Finally, to prove part (i), we first observe that

$$
\left[\mathbf{X}_{V U}\right]_{i, j}(k)=\left\langle\phi_{V}^{i}, \mathbf{O}^{k} \phi_{U}^{j}\right\rangle_{\mathcal{H}}=\overline{\left\langle\mathbf{O}^{k} \phi_{U}^{j}, \phi_{V}^{i}\right\rangle_{\mathcal{H}}}=\overline{\left\langle\phi_{U}^{j}, \mathbf{O}^{-k} \phi_{V}^{i}\right\rangle_{\mathcal{H}}}
$$

where $\bar{z}$ denotes the complex conjugate of $z$. A simple computation in Fourier domain yields

$$
\widehat{\mathbf{X}}_{U V}(f)=\widehat{\mathbf{X}}_{V U}^{*}(f),
$$


where $\widehat{\mathbf{X}}_{V U}(f)$ is the matrix associated with the oblique projection $\mathbf{P}_{V \perp U}$, and where the superscript $*$ denotes the Hermitian transpose. It follows that the operators $\widehat{\Xi}_{U V}$ and $\widehat{\Xi}_{V U}$ (see eqs. (3.10) and (3.11)) can also be written as

$$
\begin{gathered}
\widehat{\Xi}_{U V}(f)=\widehat{\mathbf{R}}_{U V}(f) \widehat{\mathbf{R}}_{U V}^{*}(f)=\widehat{\mathbf{R}}_{V U}^{*}(f) \widehat{\mathbf{R}}_{V U}, \\
\widehat{\Xi}_{V U}(f)=\widehat{\mathbf{R}}_{V U}(f) \widehat{\mathbf{R}}_{V U}^{*}(f)=\widehat{\mathbf{R}}_{U V}^{*}(f) \widehat{\mathbf{R}}_{U V}(f) .
\end{gathered}
$$

Using the last two relations, we infer that if $\lambda(f)$ is an eigenvalue for $\widehat{\Xi}_{V U}(f)$ (i.e., $\left.\widehat{\mathbf{R}}_{V U} \widehat{\mathbf{R}}_{V U}^{*} x=\lambda x\right)$, then we have that $\widehat{\mathbf{R}}_{V U}^{*} \widehat{\mathbf{R}}_{V U}\left(\widehat{\mathbf{R}}_{V U}^{*} x\right)=\lambda\left(\widehat{\mathbf{R}}_{V U}^{*} x\right)$. Hence, $\lambda$ is also an eigenvalue of $\widehat{\Xi}_{U V}(f)$. Since $\widehat{\Xi}_{V U}(f)$ and $\widehat{\Xi}_{U V}(f)$ have the same eigenvalues, we conclude from (3.9) and (3.13) that $\cos \theta(U, V)=\cos \theta(V, U)=\rho$.

If the conditions of Theorems 3.1 or 3.2 are satisfied, then the oblique projection can be written as

$$
\mathbf{P}_{U \perp V} g=\sum_{(k, l) \in \mathbf{Z}^{2}} \mathbf{G}(k) \mathbf{X}_{U V}^{i n v}(l-k) \Phi_{U}^{T}(l)
$$

where $\mathbf{X}_{U V}^{i n v}(k)$ is the inverse Fourier transform of $\widehat{\mathbf{X}}_{U V}^{-1}(f)$. The operations involved in computing the coefficients of the series (3.21) consist of simple convolutions and additions only. As previously mentioned, this feature is useful for implementation.

\section{EXAMPLES AND CONCLUDING REMARKS}

The oblique projection in shift-invariant subspaces of $L_{2}$ is implicit in the general theory of biorthogonal wavelets of A. Cohen, I. Daubechies, and J.C. Fauveau [7]. Our results can be used to extend the biorthgonal wavelet theory to spaces other than $L_{2}$. This has been done for the sequence space $l_{2}$, which is useful in digital signal processing [2]. Other extensions of interest would be to biorthogonal multiwavelets and to geometries other than the Euclidian space. In particular, mutiresolution on the sphere would be useful in geophysics.

Shift-invariant spaces have also been used to generalize the Shannon-Whittaker theory on uniform sampling [3], and the oblique projections have been used to take into account nonideal acquisition devices [19]. The present results may also be useful in multimodal signal and image processing.

\section{ACKNowledgments}

I thank Ingrid Daubechies for useful discussions and a suggestion concerning a possible application. I thank Hans Feichtinger for useful references. I also thank Barry Bowman and Mike Vrhel for their editorial help. Finally, I would like to thank Jane Bradley; why not?

\section{REFERENCES}

1. A. Aldroubi and M. Unser, Families of multiresolution and wavelet spaces with optimal properties, Numer. Funct. Anal. and Optimiz., 14:417-446, 1993. MR 94j:42045

2. A. Aldroubi and M. Unser, Oblique projections in discrete signal subspaces of $l_{2}$ and the wavelet transform, (Andrew Laine and Michael Unser, eds.), Wavelet applications in signal and image processing, SPIE-The international Society for Optical Engineering, Bellingham, WA, 1994, pp. 36-45. 
3. A. Aldroubi and M. Unser, Sampling procedure in function spaces and asymptotic equivalence with shannon's sampling theory, Numer. Funct. Anal. and Optimiz., 15:1-21, 1994. MR 95a:94002

4. J. J. Benedetto and M. W. Frazier, Wavelets-Mathematics and Applications, CRC, Boca Raton, FL, 1993.

5. C. A. Berenstein and E. V. Patrick, Exact deconvolution for multiple convolution operatorsan overview, plus performance characterizations for imageing sensors, IEEE, pages 723-734. IEEE, April 1990.

6. Carl De Boor, Ronald DeVore, and Amos Ron, Approximation from shift-invariant subspaces of $L_{2}\left(\mathbf{R}^{d}\right)$, Trans. Amer. Math. Soc., 341(2):787-806, 1994. MR 94d:41028

7. A. Cohen, I. Daubechies, and J.C. Fauveau, Biorthogonal bases of compactly supported wavelets, Comm. Pure Appl. Math., 45:485-560, 92. MR 93e:42044

8. I. Daubechies, Ten Lectures on Wavelets, SIAM, Philadelphia, PA, 1992. MR 93e:42045

9. G. Donovan and J.S. Geronimo, Fractal functions, splines, intertwining multiresolution analysis, and wavelets, (Andrew Laine and Michael Unser, eds.), Wavelet applications in signal and image processing, pages 238-243, Bellingham, Washington USA, 1994. SPIE- The international Society for Optical Engineering.

10. H. G. Feichtinger, Pseudo-inverse matrix methods for signal reconstruction from partial data, SPIE-Conf., Visual Comm. and Image Proc., Boston, Int. Soc. Opt. Eng., 1991, pp. 766-772.

11. T. N. T. Goodman, S. L. Tang, and W.S. Lee, Wavelet wandering subspaces, Trans. Amer. Math. Soc., 338(2):639-654, 1993.

12. C. Houdre, On the linear prediction of multivariate $(2, p)$-bounded processes, Ann. Probab., 19(2):843-867,1991.

13. S. Mallat, Multiresolution approximations and wavelet orthonormal bases of $L^{2}(\mathbf{R})$, Trans. Amer. Math. Soc., 315(1):69-97, 1989. MR 90e:42046

14. Y. Meyer, Ondelettes et opérateurs, Hermann, Paris, 1990.

15. C.A. Michelli, Using the refinement equation for the construction of pre-wavelet, Numerical Algorithms, 1:75-116, 1991.

16. G. Strang and G. Fix, A Fourier analysis of the finite element variational method, Edizione Cemonese, Rome, 1973, pp. 793-840.

17. V. Strela and G Strang, Finite element multiwavelets, (Andrew Laine and Michael Unser, eds.), Wavelet applications in signal and image processing, pages 202-213, Bellingham, Washington USA, 1994. SPIE- The international Society for Optical Engineering.

18. W. Sweldens and R. Piessens, Quadrature formulae and asymptotic error expansions for wavelet approximations of smooth functions, SIAM Journal on Numerical Analysis, 31(4):1240-1264, 1994. MR 95e:42043

19. M. Unser and A. Aldroubi, A general sampling theory for non-ideal acquisition devices, IEEE Trans. on Signal Processing, 42(11):2915-2925, 1994.

20. M. J. Vrhel and M. Unser, Multi-channel deconvolution: the generalized sampling approach, (Andrew Laine and Michael Unser, eds.), Wavelet applications in signal and image processing, pages 188-199, Bellingham, Washington USA, 1994. SPIE- The international Society for Optical Engineering.

NIH/BEIP, Building 13/3N17, 13 South DR MSC 5766, Bethesda, Maryland 20892-5766

E-mail address: aldroubi@helix.nih.gov 